\section{Living with the enemy}

Infection can result in two different responses by the host, one focused on elimination and the other focused on 'tolerance' (defined here as coexistence with the infection with few or no sequelae). In PLoS Biology, Begon and colleagues use a longitudinal analysis of field voles caught in the wild to examine immunological parameters, microbial burden and body condition to understand the basis of tolerance of infection. Microparasite burdens increase with age but, unexpectedly, this is associated with better condition of the animal. This improved condition is not correlated with established regulatory factors such as IL-10, TGF- $\beta$ or Foxp3 but instead is correlated with only GATA-3, a classic $T$ helper type $2\left(T_{H} 2\right)$ transcription factor. GATA-3 is also strongly predictive of host survival but at the cost of diminished fecundity. Not only does this demonstrate the immunological value of studying 'true wild-type' populations, it suggests that type 2 immunity might be understood less as a macroparasite-expulsion mechanism and more as a damage-limitation exercise. $Z F$ PLoS Biol. (8 July 2014) doi:10.1371/journal.pbio. 1001901

\section{MicroRNA and $\mathrm{T}_{\mathrm{H}} 2$ cells}

The differentiation of helper $\mathrm{T}$ cell subsets is influenced by a diversity of microRNAs. In the Proceedings of the National Academy of Sciences USA, Wilson and colleagues use a comprehensive transcriptomics approach to identify microRNA signatures associated with the $\mathrm{T}_{\mathrm{H}} 1, \mathrm{~T}_{\mathrm{H}} 2, \mathrm{~T}_{\mathrm{H}} 17$ and induced regulatory $\mathrm{T}$ cell subsets in vitro and in vivo. $\mathrm{T}_{\mathrm{H}} 2$ cells generated in vivo have a miRNA profile clearly distinguishable from that of other subsets and, unexpectedly, also show very little overlap with their $\mathrm{T}_{\mathrm{H}} 2$ counterparts generated in vitro. In particular, the microRNAs miR-155 and miR-146a are dynamically regulated in $\mathrm{T}_{\mathrm{H}} 2$ cells generated in vivo, with the former promoting and the latter regulating effector function in a cell-intrinsic manner. miR-155 regulates expression of the $\mathrm{S}_{1} \mathrm{P}_{1}$ receptor for the bioactive lipid S1P and may therefore exert its effects in part by controlling migration to sites of tissue inflammation. Targeting miR155 may therefore alleviate type 2 pathologies such as allergy. $\quad Z F$ PNAS (14 July 2014) doi:10.1073/pnas.1406322111

\section{Autophagosome formation}

Salmonella infect cells and proliferate in intracellular vacuoles. Host cell-mediated autophagy is needed to eliminate infectious bacteria, but how autophagosomes develop on these vacuoles is incompletely understood. In Molecular Cell, Tooze and colleagues show that WIPI2b, which binds phosphatidylinositol-3-phosphate, is needed to recruit the autophagy protein Atg16L1 to the salmonellacontaining vacuole. Atg16L1, in complex with Atg5 and Atg12, conjugates the marker LC3 to the lipid membrane of the developing autophagosome. Replacement of the glutamic acid residue Glu226 or Glu230 in Atg16L1 or the arginine residue Arg108 or Arg125 in WIP12b abolishes this interaction and autophagosome formation. Thus, WIP2b serves as a bridge that links the generation of phosphatidylinositol-3-phosphate on bacteria-containing vacuoles to their destruction by autophagy.

Mol. Cell 55, 238-252 (2014)

\section{Regulating gut peristalsis}

Intestinal peristalsis is regulated by the enteric nervous system and is influenced by dietary and microbial changes. In Cell, Muller et al. show that a distinct population of macrophages that are distributed in the intestinal smooth muscles (muscularis macrophages) and are characterized as MHCII ${ }^{\text {hi }} \mathrm{CD} 11 \mathrm{c}^{\mathrm{lo}} \mathrm{CD} 103^{-} \mathrm{CD} 11 \mathrm{~b}^{+} \mathrm{CX} 3 \mathrm{CR} 1^{\text {hi }}$ cells regulate the peristaltic activity of the colon in the steady state. Muscularis macrophages are found along nerve fibers and secrete the soluble mediator BMP2, which signals constitutively to enteric neurons through the BMP receptor. In turn, enteric neurons contribute to the maintenance of muscularis macrophages through secretion of the macrophage growth factor CSF1. Antibiotic treatment diminishes the expression of BMP2 and the number of macrophages, as well as signaling via the BMP receptor and expression of CSF1 in neurons and results in dysregulated peristalsis. These findings suggest that the macrophage-neuron crosstalk that controls gut peristalsis is influenced by bacterial commensals.

Cell 158, 300-313 (2014)

\section{Memory stemness}

It remains unclear if long-term immunological memory is maintained through stem cell-based-type mechanisms. In Immunity, Graef et al. investigate the multipotency, self-renewal and immune system-reconstituting ability of individual memory $T$ cells through serial single-cell adoptive transfer and infectioninduced population reexpansion. Single $\mathrm{CD} 44^{\mathrm{hi}} \mathrm{CD} 62 \mathrm{~L}^{+}$central memory $T$ cells $\left(\mathrm{T}_{\mathrm{CM}}\right.$ cells) derived from transferred naive ovalbumin-specific OT-I T cells and sorted at least 100 days after infection with ovalbumin-expressing Listeria monocytogenes generate immune responses of a size, stochastic variation and phenotype (effector T cell, effector-memory $\mathrm{T}$ cell or $\mathrm{T}_{\mathrm{CM}}$ cell) similar to that generated by single naive $\mathrm{T}$ cells after secondary transfer. After repetitive single-cell adoptive transfer and infection-driven population reexpansion, primary and secondary $\mathrm{T}_{\mathrm{CM}}$ cells show broad proliferation and differentiation potential similar to that of naive $\mathrm{T}$ cells, and the tertiary progeny of two distinct secondary $T_{C M}$ cells are diverse and distinct. These results suggest that individual $\mathrm{T}_{\mathrm{CM}}$ cells have true self-renewal ability and are multipotent.

Immunity 41, 116-126 (2014)

\section{Tumor-macrophage interactions}

How tumor cells communicate with tumor-associated macrophages (TAMs) to influence their function is not known. In Nature, Colegio et al. report that tumor-cell production of lactic acid via glycolysis pathways acts on TAMs to upregulate their expression of genes encoding the the growth factor VEGF and the arginase Arg1. Inhibition of monocarboxylate transporters, which blocks the uptake of lactate acid, prevents macrophage expression of Vegf. Lactic acid somehow stabilizes the transcription factor HIF- $1 \alpha$, which then positively regulates the expression of a subset of genes of M2-polarized macrophages. Notably, this response is independent of interleukin 4 (IL-4) or IL-13, cytokines commonly associated with the M2 polarization of macrophages. Macrophage-specific conditional deletion of Hifla or Arg1 in a tumor model leads to a diminished tumor burden. Thus, bidirectional communication is established between tumor cells and TAMs that is beneficial for tumor growth.

Nature (13 July 2014) doi:10.1038/nature13490
$L A D$ 